\title{
Soft QCD Dynamics of Elastic Scattering in Impact Parameter Representation
}

\author{
B.Z. Kopeliovich ${ }^{1,2}$, I.K. Potashnikova ${ }^{1,2}$, B. Povh ${ }^{1}$ and E. Predazzi ${ }^{3}$ \\ ${ }^{1}$ Max-Planck Institut für Kernphysik, Postfach 103980, 69029 Heidelberg, Germany \\ ${ }^{2}$ Joint Institute for Nuclear Research, Dubna, 141980 Moscow Region, Russia \\ ${ }^{3}$ Università di Torino and INFN, Sezione di Torino, I-10125, Torino, Italy
}

\begin{abstract}
The elastic hadronic amplitude is calculated using the nonperturbative light-cone dipole representation for gluon bremsstrahlung. The data for large mass diffraction demand a two-scale structure of light hadrons: the gluon clouds of the valence quarks with the size of $\sim 0.3 \mathrm{fm}$ and the hadronic size $\sim 1 \mathrm{fm}$. The presence of the two scales unavoidably leads to a specific form for the total hadronic cross section which consists of a steeply rising $\propto s^{\Delta}(\Delta=0.17 \pm 0.01)$ term related to gluon radiation, and a large constant term originating from soft interactions which does not induce any gluon emission. Our calculations reproduce well the total cross sections and elastic slopes [1]. To further test the model, we analyze the elastic $p p$ and $\bar{p} p$ differential cross sections and extract the partial amplitudes in the impact parameter representation. The Pomeron trajectory as a function of the impact parameter is only slightly above one for central collisions, but steeply grows towards the periphery. The model predicts correctly the shape and energy dependence of the partial amplitude at all impact parameters.
\end{abstract}




\section{Introduction}

\subsection{Rising total cross sections}

The increase of the total hadronic cross section at high energies is well known since the ISR experiments in the early 70's. This discovery came just in the time when the Regge theory had its conjecture. The simple idea to shift the intercept of the Pomeron pole above one, $\alpha_{P}(0)=1+\Delta$, leads to contradiction with the unitarity restrictions, in particular, the Froissart bound is violated. It took special efforts to formulate a self-consistent Regge scheme [2] in which unitarity is restored via Regge cuts and without violation of energy conservation (warnings of which had been given in [3]).

The assumption that the Pomeron which governs the hadronic elastic amplitude at high energies is a Regge pole has no theoretical justification beyond simplicity. It faces problems interpreting data from HERA which demonstrate that $\Delta$ substantially increases with $Q^{2}$ (see, however, [4], [5]).

The ensemble of data on hadronic elastic scattering at small $t$, i.e. total and differential cross sections, slopes and ratios of real to imaginary part of the forward elastic amplitudes can successfully be fitted by many phenomenological models based on varieties of quite different assumptions aimed to fit the data (see, for example [6]). Even the simple parameterization $s^{\Delta}$ with exponential $t$-dependence for the elastic amplitude describes well the data at small $t[0,8]$. However, the imaginary part of the forward elastic amplitude is connected by the unitarity relation to the total inelastic cross section, in other words, the Pomeron is the shadow of inelastic processes. Unavoidably, one should study the dynamics of inelastic collisions to understand the forward elastic scattering [3], rather than guessing its analytic form which is only mildly restricted by general principles.

The total cross section for a highly virtual photon interacting with a proton measured in deep-inelastic lepton scattering (DIS) can be estimated using perturbative QCD if the

photon virtuality $Q^{2} \gg Q_{0}^{2}\left(Q_{0} \sim 1 G e V\right)$ and the energy $s \gg s_{0}\left(s_{0} \sim 1 G e V^{2}\right)$, but 
$x=Q^{2} / s \ll 1$. Depending on the approximations used, two models for the hard Pomeron are known: the BFKL [9] and the double-leading-log DGLAP (see in [10, 11]). The rising energy dependence is interpreted perturbatively as caused by gluon bremsstrahlung with growing phase space for radiated gluons. The total cross section is predicted to rise steeply with energy as is confirmed by data from HERA. The energy dependence, parameterized as $s^{\Delta_{e f f}\left(Q^{2}\right)}$ reveals the exponent to increase with $Q^{2}$ up to $\Delta_{e f f} \sim 0.5$.

In terms of the QCD light-cone dipole approach one can treat DIS at small $x$ as an interaction of a tiny size, $\sim 1 / Q$, quark-antiquark fluctuations surrounded by a gluon cloud which is much larger (logarithmically) than the $\bar{q} q$ pair.

\subsection{Soft interaction limit: the two scales for light hadrons}

A new scheme for performing explicit calculations for the interaction of light hadrons has been suggested in [1]. It exploits the smallness of the gluon correlation radius which has been estimated in many approaches. In particular, the model developed in ref. [12], extends the perturbative methods of the light-cone QCD to the nonperturbative region introducing the light-cone potential into the Schrödinger equation for the Green function that describes the propagation of a quark-gluon interacting pair. The interaction potential fixed by the data for large mass soft diffraction turns out to be rather strong leading also to a short separation $r_{0}=0.3 \mathrm{fm}$ between gluons and the source (a quark or a gluon). This result is confirmed by the recent analysis [13] of HERA data for diffraction which leads to an even smaller estimate $r_{0} \approx 0.2 \mathrm{fm}$ (but with large uncertainties). Thus, a proton looks in the infinite momentum frame like three valence quarks surrounded by small gluon clouds as illustrated in Fig. 11.

Such a two-scale structure of light hadrons appears not only in the model [12] treating the vacuum fluctuations as Weizsäker-Williams gluons. The smallness of the gluon clouds of the valence quarks is confirmed by the study of the gluon formfactor of the proton employing QCD sum rules [14]. The $Q^{2}$ dependence of the formfactor turns out to be rather weak 


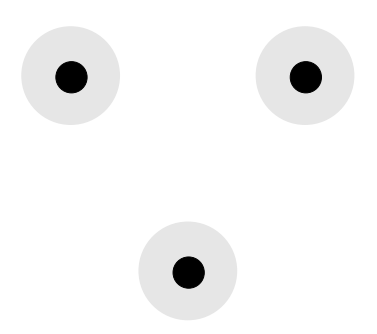

Figure 1: A skeleton of three valence quarks in the proton, surrounded by gluon clouds of much smaller size than the mean quark separation.

corresponding to a small radius of the gluon distribution which was estimated at the same value $r_{0} \approx 0.3 \mathrm{fm}$. The small gluon correlation radius $\sim 0.3 \mathrm{fm}$ appears also from lattice calculations [15]. It is also predicted by the liquid instanton model [16, 17] and is related to the instanton size $\rho_{0} \sim 0.3 \mathrm{fm}$. The experimental observation of a small cross section for large mass soft diffractive dissociation has led to a small value of $r_{0}$ [12, [13] and this, quite in general, can be taken as the confirmation of the small size cloud of any kind of gluonic vacuum fluctuations surrounding the valence quarks. These are usually referred to as constituent quarks although nothing specific (about mass, additivity, etc.) is assumed beyond the simple statement that the clouds of vacuum fluctuations dressing the valence quarks are much smaller than the mean hadronic radius.

Of course the transverse size of the gluonic spots increases with energy since the weights of higher Fock components grow as powers of $\ln (s)$ [1, 17]. Such a behavior is specific of gluonic fluctuations. Nevertheless, the mean size of the fluctuation clouds is still small compared to the radii of light hadrons in the energy range of modern accelerators (see [1] and below). The ratio of the constituent quark radius $r_{0}$ to the mean interquark separation $R_{h}$ squared serves as a small number. Correspondingly, one should single out two different contributions to the total inelastic cross sections.

The first one is due to the soft interaction which is unable to resolve the structure of the constituent quarks and excite them. This contribution can be treated as the cross 
section of interacting hadrons made of structureless valence quarks which can be viewed as the skeleton of the hadrons. One can try to evaluate it using either the naive twogluon exchange approximation [18, 19, 20, 21, 17, 22] or more sophisticated nonperturbative approaches like string crossing and rearrangement [23], or the interaction of overlapping Wilson loops in the stochastic vacuum model [24, 25]. This part of the cross section $\sigma_{0}\left(R_{h}\right)$ is controlled by the mean interquark separation $R_{h}$ and is independent of energy since the size of the quark skeleton of the hadron is constant.

The second contribution to the total cross section comes from the semi-hard interaction able to resolve the small size of the constituent quarks and to excite them giving origin to gluon radiation. This cross section is proportional to the size of the constituent quark, $\propto r_{0}^{2}$. The radiation of each new gluon leads as usual to an extra power of $\ln (s)$ which exponentiates to a $\sigma_{1}\left(r_{0}\right) s^{\Delta}$ dependence. However, the energy independent term $\sigma_{1}\left(r_{0}\right)$ needed for the exponentiation of these logs is rather small $\left(\propto r_{0}^{2}\right)$ and can not match the large term $\sigma_{0}\left(R_{h}\right)$.

Thus, we arrive at the following general structure of the total cross section which corresponds to a two-scales scheme for the hadronic structure,

$$
\sigma_{t o t}=\tilde{\sigma}_{0}+\sigma_{1}\left(r_{0}\right)\left(\frac{s}{s_{0}}\right)^{\Delta},
$$

where $\tilde{\sigma}_{0}=\sigma_{0}\left(R_{h}\right)-\sigma_{1}\left(r_{0}\right)$. Parametrically, $\sigma_{0}\left(R_{h}\right) \gg \sigma_{1}\left(r_{0}\right)$.

The double scale structure of light hadrons, (1.e. small constituent quarks versus large interquark separation), leads to the structure of the total cross section Eq. (11), rather more complex than the usually assumed overall behavior $\propto s^{\Delta}$. Of course, in the spirit of the leading- $\log (\mathrm{s})$ approximation, one can neglect the constant term $\sigma_{0}$ as $s \rightarrow \infty$, but then $\Delta$ should not be compared with the experimental data available in the energy range where $\sigma_{0}$ gives an important contribution. The effective slope of the energy dependence may, in fact, be substantially smaller,

$$
\Delta_{e f f}=\left(1-\frac{\tilde{\sigma}_{0}}{\sigma_{t o t}}\right) \Delta
$$

In fact, in [1] it is found that $\Delta_{e f f} \approx \Delta / 2$. 


\subsection{Outline of the paper}

This paper is organized as follows. In section 2 we calculate the cross section of gluon radiation in high energy hadronic interactions. It is shown that valence quarks contribute additively to the radiation cross section due to the short-range correlation of the radiated gluons. Moreover, the sum of the multigluon radiation cross sections which depend on energy as powers of lns exponentiates in the leading-log approximation to the energy dependent power $s^{\Delta}$. The exponent proportional to the running QCD coupling $\alpha_{s}$ turns out to be rather large, $\Delta=0.17 \pm 0.01$ compared to what is believed to be demanded by the present data for total cross section. However, gluon radiation contributes with a rather small factor proportional to $r_{0}^{2} \approx 1 \mathrm{mb}$. This energy dependent fraction of the total cross section is fully predicted. The large energy independent part of the cross section is due to the interaction of the valence quarks with no gluon radiation. This cross section is related to the large hadronic size, rather than to $r_{0}$, and cannot be evaluated perturbatively. Although it can be estimated in models, e.g. as it is done in the stochastic vacuum model [24, 25], the uncertainty of such calculations is too large, and we prefer to treat $\tilde{\sigma}_{0}$ as a free parameter, which turns out to be the only unknown of the model.

The rising total cross section eventually violates the Froissart-Marten bound at very high energies, but the partial elastic amplitude at small impact parameters is already very close to the limit imposed by unitarity and may easily break it down. The procedure of unitarization of the elastic partial amplitudes is described in section 3. We use the standard quasi-eikonal model, but we compare also with a different QCD motivated approach.

The model is analyzed with respect to the total cross section data in section $⿴$. The only parameter of the model, $\tilde{\sigma}_{0}$, can be fixed by comparison with the data at any chosen energy. Then, the energy dependence is predicted in a good agreement with the data. The slope of forward elastic scattering needs no new parameters and is also well predicted.

In the standard Regge phenomenology the energy dependence of the total cross sections and of the elastic slopes are controlled by the intercept and slope of the Pomeron trajectory, 
respectively, which are independent parameters. However, one may expect them to be correlated since in QCD the cross sections depend on the hadronic sizes due to color screening [18, 19, 20, 26]. An attempt to incorporate this property was made recently in [27]. Here we develop this approach treating more consistently the phase space for the radiated gluons.

The comparison with the data turns out to be most effective in the impact parameter representation. First of all, the radius of interaction exposes explicitly in this case. Secondly, unitarity imposes severe restrictions on the elastic partial amplitude for central hadronic collisions [28], which slows down the energy dependence of the partial amplitude [29, 28]. Thirdly, the color dipole representation in QCD introduced in 30 became a popular tool to study high energy QCD dynamics in DIS, Drell-Yan reaction etc., since color dipoles are the eigenstates of the interaction at high energies. In this respect, the impact parameter representation is suitable for a direct comparison of the data with a dynamical model (see for instance [31]). And, last but not least, the shape of the amplitude in the impact parameter space is related to the shape of the amplitude as function of momentum transfer in a wide range of $t$, rather than only in the forward direction.

In section 5 we analyze the available high energy data from $I S R$ and $S \bar{p} p S$ for $p p$ and $\bar{p} p$ elastic scattering to extract the partial elastic amplitude in the impact parameter representation. We follow the procedure suggested by Amaldi and Schubert [29] who performed a similar analysis of ISR data and concluded that the total cross section rises due to peripheral interactions while the partial amplitude for central collisions is energy independent. This is usually treated as a manifestation of unitarity saturation. However, the parameterization of the amplitude used in 229 was based on the geometrical scaling model which assumes that the ratio of the total cross section to the elastic slope is independent of energy. This assumption unavoidably leads to a constant partial amplitude at zero impact parameter (see Fig. 9).

The geometrical scaling is known to be broken beyond the ISR energy range, therefore we perform the analysis differently, in a less model dependent way. We fit the $t$-dependence of the cross section independently at each energy assuming no correlation between different 
energies, except the normalization which is adjusted to fit the energy dependent total cross section and ratio of real to imaginary parts of the forward elastic amplitude. The $t$-dependent imaginary part of the amplitude arising from the fit is then Fourier transformed to the impact parameter representation at each energy. The $b$-dependence of the partial amplitudes found this way is very close to what our model predicts. Not only the shape of the partial amplitude is well reproduced, but also its development as function of energy.

In section 6 we compare the data for the partial amplitudes at different energies and conclude that they hardly vary at $b \approx 0$, but rise steeply with energy at large $b>1 \mathrm{fm}$. The effective Pomeron trajectory is a steeply rising function of the impact parameter. Our model correctly predicts this dependence.

The results of the paper are summarized in section 7. Further evidences of the large value of $\Delta$ suggested by data on diffraction in DIS and particle production at mid rapidities in soft hadronic collisions are reviewed.

\section{Excitation of valence quarks: nonperturbative gluon radiation}

To calculate the energy dependent total cross section one should sum up the various contributions of different Fock components of the incoming hadron. To avoid double-counting, we sum the cross sections $\sigma_{n}$ of physical process of radiation of $n$ gluons,

$$
\sigma_{t o t}^{h N}=\sum_{n} \sigma_{n}^{h N}
$$

The lowest Fock component $(n=0)$ of a hadron contains only valence quarks. For the sake of simplicity we assume the beam hadron to be a meson, the generalization to a nucleon is simple and is done below.

The contribution to the total cross section corresponding to the interaction without any 
gluon radiation has the form,

$$
\sigma_{0}^{h N}=\int_{0}^{1} d \alpha_{q} \int d^{2} R\left|\Psi_{\bar{q} q}^{h}\left(\alpha_{q}, R\right)\right|^{2} \sigma_{\bar{q} q}^{N}(R) .
$$

Here the valence quark wave function of the hadron, $\Psi_{\bar{q} q}^{h}\left(\alpha_{q}, R\right)$, depends on the transverse $q-\bar{q}$ separation $R$ (see Fig. 2) and the fraction $\alpha_{q}$ of the light-cone momentum of the pair

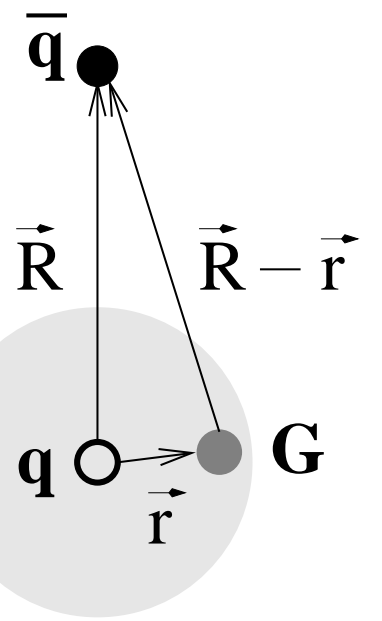

Figure 2: A cartoon for the Fock state $|\bar{q} q G\rangle$ in the impact parameter plane.

carried by the quark. The energy independent Born cross section of interaction of a large $\bar{q} q$ dipole with a nucleon $\sigma_{\bar{q} q}^{N}(R)$ cannot be calculated perturbatively since the separation $R$ is large. It can not be adjusted directly to the experimental data since the data include the contribution from gluon bremsstrahlung leading to the energy dependence of $\sigma_{\bar{q} q}^{N}$. Instead, we treat $\sigma_{0}^{h N}$ as a free parameter.

The next contribution to the $\sigma_{\text {tot }}^{h N}$ comes from radiating a single gluon. The radiation is possible only due to the difference between the amplitudes for the $\bar{q} q$ and $\bar{q} q G$ Fock components, otherwise the interaction does not alter the combination of Fock states and they remain coherent, i.e. nothing new is produced. Another way to explain this is to say that the interaction with the target can free the gluon fluctuation only if it resolves it, i.e. discriminates between the interaction amplitudes for two Fock components $|\bar{q} q\rangle$ and $|\bar{q} q G\rangle$. 
The contribution to the total cross section corresponding to radiating a single gluon reads [12, [1],

$$
\begin{aligned}
\sigma_{1}^{h N} & =\int_{0}^{1} d \alpha_{q} \int d^{2} R\left|\Psi_{\bar{q} q}^{h}\left(R, \alpha_{q}\right)\right|^{2} \int_{\alpha_{G} \ll 1} \frac{d \alpha_{G}}{\alpha_{G}} \int d^{2} r \\
& \times \frac{9}{4}\left\{\left|\Psi_{\bar{q} G}\left(\vec{R}+\vec{r}, \alpha_{G}\right)\right|^{2} \sigma_{\bar{q} q}^{N}(\vec{R}+\vec{r})+\left|\Psi_{q G}\left(\vec{r}, \alpha_{G}\right)\right|^{2} \sigma_{\bar{q} q}^{N}(r)\right. \\
& \left.-\operatorname{Re} \Psi_{q G}^{*}\left(\vec{r}, \alpha_{G}\right) \Psi_{\bar{q} G}\left(\vec{R}+\vec{r}, \alpha_{G}\right)\left[\sigma_{\bar{q} q}^{N}(\vec{R}+\vec{r})+\sigma_{\bar{q} q}^{N}(r)-\sigma_{\bar{q} q}^{N}(R)\right]\right\}
\end{aligned}
$$

Here $\alpha_{G}$ is the fraction of the hadron momentum carried by the gluon which is assumed to be small; the notations for the radii are obvious from Fig. 2. The first and the second terms in the curly brackets correspond to the emission of the gluon from the quark and the antiquark respectively, and the third term to the interference between them.

The nonperturbative wave function for a quark-gluon Fock component was derived in [12. Neglecting the quark mass the wave function reads,

$$
\left.\Psi_{q G}\left(\vec{r}, \alpha_{G}\right)\right|_{\alpha \ll 1}=-\frac{2 i}{\pi} \sqrt{\frac{\alpha_{s}}{3}} \frac{\vec{e}^{*} \cdot \vec{r}}{r^{2}} \exp \left(-\frac{r^{2}}{2 r_{0}^{2}}\right),
$$

where $\vec{e}$ is the polarization vector of the massless gluon. The mean separation $r_{0}=0.3 \mathrm{fm}$ is related to the nonperturbative light-cone potential describing the quark gluon interaction. It is fixed by the data on large mass diffractive dissociation corresponding to the triplePomeron limit. The mean quark - gluon separation $r_{0}$ is much smaller than the distance $R$ between the quarks. Therefore, one of the $q G$ wave functions in (5) can be neglected leaving a factor 2 (a factor 3 in the case of $N N$ scattering) since both $q$ and $\bar{q}$ can radiate the gluon.

At small separations, $r \sim r_{0}$, the dipole cross section $\sigma_{\bar{q} q}^{N}(r)$ can be evaluated perturbatively and the approximation $\sigma_{\bar{q} q}^{N}(r)=C r^{2}$ can be used. The two-gluon approximation gives for the factor $C \approx 2.3 \mathrm{using}$ an effective gluon mass $m_{G}=0.15 \mathrm{GeV}$ to incorporate confinement, and $\alpha_{s}=0.4$ (see below).

Thus, the contribution of the $|\bar{q} q G\rangle$ Fock component to the total cross section summed over polarization of the radiated gluon takes the form,

$$
\sigma_{1}^{h N}=\frac{4 \alpha_{s}}{3 \pi} \ln \left(\frac{s}{s_{0}}\right) \frac{9}{4} C r_{0}^{2}
$$


Here $\ln \left[s / s_{0}\right]=\ln \left[\left(\alpha_{G}\right)_{\max } /\left(\alpha_{G}\right)_{\min }\right]$ originates from the integration over $\alpha_{G}$ in (5), where $\left(\alpha_{G}\right)_{\min }=2 / s r_{0}^{2} \approx\left(1 G e V^{2}\right) / s$, but $\left(\alpha_{G}\right)_{\max }$ is ill defined. It should be small enough, say $\sim 0.1$ to make sure that the quark-gluon wave functions in (5) is independent of $\alpha_{G}$. Then, assuming that the quark carries a fraction one third of the proton momentum, we estimate the value $s_{0} \sim 30 \mathrm{GeV}$, which we use in what follows.

The radiation of each new, $n$-th, gluon can be treated as the radiation by an effective quark, which is the valence quark surrounded by $n-1$ gluons. It should be resolved by the soft interaction with the target as being different from the radiation of $n-1$ gluons. Therefore, it provides the same mean cross section $9 C r_{0}^{2} / 4$ and the factor $4 \alpha_{s} / 3 \pi$ as in (7). This is illustrated in Fig. 3 in the $1 / N_{c}$ approximation, i.e. replacing each gluon by a $\bar{q} q$ pair. According to the general prescription [32, 12] of the light-cone approach, the

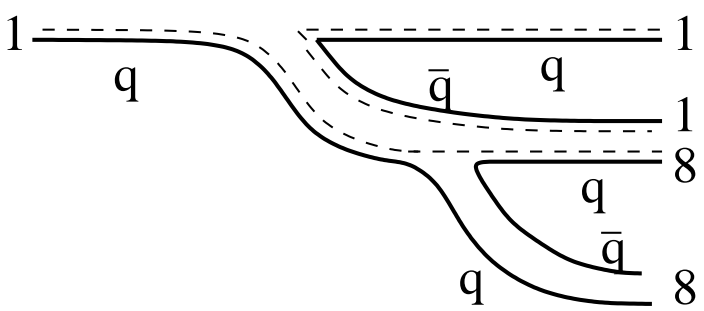

Figure 3: Radiation of the second gluon in the leading-log(s) approximation as seen in $1 / N_{c}$ approximation when each gluon is replaced by a $\bar{q} q$ pair. Solid quark lines correspond to the final state, dashed - lines correspond to the initial state configurations.

radiation cross section in the impact parameter representation is proportional to the total cross section of a colorless system made of all the final state partons (solid lines in Fig. 3) plus the initial state partons replaced by antipartons (dashed lines). Since the radiation of gluons with $\alpha_{G} \ll 1$ does not affect the impact parameter of the radiating quark, all the solid and the corresponding dashed lines have the same impact parameters. Therefore, those quark-antiquark (solid-dashed) pairs which are color neutral do not contribute to the cross section. The only quark contributing is the one that radiates the last gluon and that 
changes color in a color-octet state with the antiquark as shown in Fig. 3. Thus, the total cross section of the multiquark configuration is reduced to one for the octet-octet dipole with mean separation $r_{0}$, i.e. $9 C r_{0}^{2} / 4$.

In conclusion, the $n$-th term in (3) reads (for a single valence quark),

$$
\sigma_{n}^{q N}=\frac{1}{n !}\left[\frac{4 \alpha_{s}}{3 \pi} \ln \left(\frac{s}{s_{0}}\right)\right]^{n} \frac{9}{4} C r_{0}^{2}
$$

Summing up the powers of logarithms in (3) we arrive at the following expression for the total cross section,

$$
\sigma_{t o t}^{p p}=\tilde{\sigma}_{0}^{p p}+3 \frac{9}{4} C r_{0}^{2}\left(\frac{s}{s_{0}}\right)^{\Delta}
$$

where

$$
\begin{gathered}
\Delta=\frac{4 \alpha_{s}}{3 \pi} . \\
\tilde{\sigma}_{0}^{p p}=\sigma_{0}^{p p}-\frac{9}{4} C r_{0}^{2} .
\end{gathered}
$$

Since each of three valence quarks can radiate (see (5)) the second term in (9) acquires factor 3 .

The structure of Eq. (9) reflects the physical input illustrated in Fig. 1, as discussed in the Introduction (section 1.2). The energy dependence of the cross section is related to the excitation of the small spots (constituent quarks) inside the hadron, while a large energy independent contribution corresponds to the soft interaction of the valence quarks skeleton of the hadrons leading to no gluon radiation. These two parts of the cross section cannot match to provide a power dependence, $s^{\Delta}$, as a common factor, as it is usually assumed to the the case in the so-called soft Pomeron approach [7, 21, 22]. This is the origin of the more complicated form we find i.e. of the structure of eq. (9) or, which is the same, of eq. (11). The energy dependent part of the cross section, i.e. the second term in (9), is suppressed by the smallness of $r_{0}^{2}$ and is expected to be relatively small at medium-high energies, but grows more steeply with energy than the overall $\sigma_{\text {tot }}^{p p}$. Note that such a structure of the total cross section was also suggested in [33], however with a different physical motivation. It was found to fit well the data on total cross section (see below). 
The power $\Delta$ in (9) is related to the Pomeron intercept, $\alpha_{P}(0)=1+\Delta$, and can be predicted using (10) provided the QCD coupling $\alpha_{s}$ at virtuality $\sim 1 / r_{0}$ is known. In Gribov's theory of confinement [34, 35] the radius of a constituent quark is at the borderline between the perturbative and the nonperturbative QCD regimes. At larger distances, the

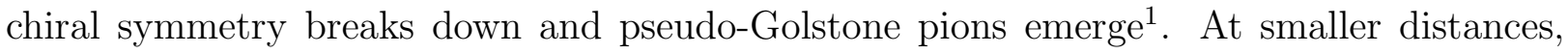
perturbative QCD is at work. The corresponding critical value of $\alpha_{s}$ is [34, 35],

$$
\alpha_{c}=\frac{3 \pi}{4}\left(1-\sqrt{\frac{2}{3}}\right) \approx 0.43 .
$$

Another way to evaluate $\alpha_{s}$ is to average the running QCD coupling weighted with the transverse momentum distribution of radiated gluons,

$$
\left\langle\alpha_{s}\right\rangle=\frac{\int_{0}^{\infty} d k_{T}^{2} \alpha_{s}\left(k_{T}^{2}\right) \frac{d \sigma(q N \rightarrow q G X)}{d\left(\ln \alpha_{G}\right) d k_{T}^{2}}}{\int_{0}^{\infty} d k_{T}^{2} \frac{d \sigma(q N \rightarrow q G X)}{d\left(\ln \alpha_{G}\right) d k_{T}^{2}}}
$$

where the transverse momentum distribution of gluons radiated in quark-nucleon interaction is given by Eq. (130) of [12].

The standard phenomenological way to extend $\alpha_{s}\left(k_{T}\right)$ down to small values $k_{T} \rightarrow 0$ is to make a shift in the argument, $k_{T}^{2} \Rightarrow k_{T}^{2}+k_{0}^{2}$. The value $k_{0}^{2} \approx 0.25 G e V^{2}$ was estimated in [35] using dispersion techniques [37] of higher twist effects in hard reactions.

We evaluated (13) using the dipole cross section $\sigma_{\bar{q} q}(\rho) \propto 1-\exp \left(-\rho^{2} / \rho_{0}^{2}\right)$ which is proportional to $\rho^{2}$ at small $\rho$, but levels off at large separations. The nonperturbative quark-gluon interaction taken into account in [12] is very important, since it squeezes the quark-gluon fluctuations down to a mean size $r_{T} \sim r_{0}$ substantially increasing the mean transverse momenta of radiated gluons. Correspondingly, the mean value $\left\langle\alpha_{s}\right\rangle$ turns out to be rather small. For the parameter $\rho_{0}$ varying within a reasonable interval $0.3<\rho_{0}<1 \mathrm{fm}$ we found the mean coupling varying between $\left\langle\alpha_{s}\right\rangle=0.38-0.43$ which agrees well with the critical value Eq. (12). Substituting the central value of $\left\langle\alpha_{s}\right\rangle$ into (10) and using the interval

\footnotetext{
${ }^{1}$ The Pomeron properties at large distances related to pion loops have been employed in the model suggested in [36]
} 
as the uncertainty for $\alpha_{s}$ we get,

$$
\Delta=0.17 \pm 0.01
$$

This value is about twice as large as the value 0.08 usually believed to be required by data. This value, however, can not be compared directly with the soft Pomeron intercept where it is assumed that the whole cross section is proportional to $s^{\Delta}$. The value of (14) is just the second term of (9) and the overall energy dependence is much less steep as a consequence of the large value of the constant term $\tilde{\sigma}_{0}^{p p}$. We will show later (see Section 凹) that the predicted energy dependence of the cross section (9) is in a good accord with the data and corresponds to an effective value $\Delta_{e f f} \approx 0.1$. First of all, however, we should take care of unitarity since the cross section (9) violates the Froissart bound at large $s$.

\section{Impact parameter representation, unitarization}

Although the total cross section stays well below the Froissart-Martin bound up to the present highest energies, the partial amplitude at small impact parameters demonstrates a precocious onset of the unitarity restrictions which are already important in the energy range of existing accelerators.

The imaginary part of the partial amplitude corresponding to the total cross section (3) can be decomposed into the terms related by unitarity to the radiation of different number $n$ of gluons,

$$
\operatorname{Im} \gamma_{P}(s, b)=\sum_{n} \operatorname{Im} \gamma_{n}(s, b)
$$

where $\gamma_{n}(s, b)$ is the partial elastic amplitude which depends on the energy and on the impact parameter $b$. Upon performing the Fourier transform of the $t$-dependent elastic amplitude, the integral over $\vec{b}$ gives the corresponding term $\sigma_{n}^{h N}$ in (3). We assume that the $t$-dependence of the lowest Fock component which is related to the spatial distribution of valence quarks is given by the product of the electromagnetic formfactors of the colliding

hadrons (we confine our considerations to $p p$ and $\bar{p} p$ collisions) $F_{p}^{2}(t)$. For simplicity we use 
the standard dipole form for the proton formfactor $F_{p}(t)=\left(1-t\left\langle r_{c h}^{2}\right\rangle / 12\right)^{-2}$, where $\left\langle r_{c h}^{2}\right\rangle$ is the mean charge radius squared related to the slope of elastic scattering of the valence quark skeleton by $B_{0}=2\left\langle r_{c h}^{2}\right\rangle / 3$.

We keep the same $t$-dependence for higher Fock components in (3) corresponding to gluon radiation by a projectile valence quark interacting with the target proton; the slope of these components, however, should increase linearly with the number of radiated gluons due to their random walk in the impact parameter plane with a step $\sim r_{0}^{2}$ for the radiation of every new gluon,

$$
B_{n}=\frac{2}{3}\left\langle r_{c h}^{2}\right\rangle+\frac{n r_{0}^{2}}{2}
$$

The Fourier transform of the square of the dipole formfactor leads to the following shape for the partial amplitudes [26],

$$
\operatorname{Im} \gamma_{n}^{p p}(b, s)=\frac{\sigma_{n}^{h N}(s)}{8 \pi B_{n}} y^{3} K_{3}(y)
$$

where $y^{2}=\left(4 b^{2} / B_{n}\right)^{3}, K_{3}(y)$ is the third order modified Bessel function and $\sigma_{n}^{h N}$ are given by (4) and (8). The normalization of the partial amplitude is fixed by the relation, $\sigma_{\text {tot }}=$ $2 \int d^{2} b \operatorname{Im} \gamma(b, s)$.

The partial amplitude (15) rises with energy and eventually would lead to a violation of the unitarity bound $\operatorname{Im} \gamma(s, b) \leq 1$, unless unitarity corrections are introduced. Unfortunately, this is not a well defined procedure since different recipes can be found in the literature.

The simplest known way to restore unitarity is to eikonalize the partial amplitude (15),

$$
\operatorname{Im} \Gamma_{P}(b, s)=1-\exp \left[-\operatorname{Im} \gamma_{P}(b, s)\right]
$$

At very high $s$ this amplitude approaches the black disk limit [2], $\operatorname{Im} \Gamma_{P}(s, b) \rightarrow \Theta\left[R^{2}(s)-b^{2}\right]$, with radius, $R(s)=r_{0} \Delta \ln \left(s / s_{0}\right)$. Correspondingly, at high energies

$$
\Delta \ln \left(\frac{s}{s_{0}}\right) \gg \frac{\left\langle r_{c h}^{2}\right\rangle}{r_{0}^{2}}
$$


all hadronic cross sections reach the maximal universal energy growth allowed by FroissartMartin's bound,

$$
\sigma_{t o t}^{h N}(s) \rightarrow 2 \pi r_{0}^{2} \Delta^{2} \ln ^{2}\left(\frac{s}{s_{0}}\right)
$$

The eikonalization procedure (18) would be suitable if the the incoming hadrons were eigenstates of the interaction [30]. Hadrons, however, are subject to diffractive off-diagonal excitation, and the eikonal form of unitarization should be corrected in a way similar to Gribov's inelastic corrections [38] for hadron-nucleus cross sections. The lowest order unitarity correction in (18) comes from the quadratic term in the exponent expansion of $\Gamma(b, s)$. It has to be modified using the AGK cutting rules [3] to include single diffraction,

$$
\operatorname{Im} \Gamma_{P}=\operatorname{Im} \gamma_{P}-\frac{1}{2}\left(\operatorname{Im} \gamma_{P}\right)^{2}[1+D(s)]+O\left(\gamma_{P}^{3}\right)
$$

where $D(s)=\sigma_{s d}(s) / \sigma_{e l}(s)$ is approximately 0.25 in the ISR energy range and decreases slightly with energy $\propto s^{-0.04}$ [39, 40]. Indeed, $\sigma_{e l}(s)=\sigma_{\text {tot }}^{2}(s) /\left(16 \pi B_{e l}\right) \propto s^{0.1}$, but the energy dependence of the diffractive cross section is rather flat (due to stronger unitarity corrections), $\sigma_{s d}(s) \propto s^{0.06}$. Asymptotically, as $s \rightarrow \infty, D(s)$ vanishes since $\sigma_{e l}(s) \propto \ln ^{2} s$ and $\sigma_{s d}(s) \propto \ln s$.

The inelastic corrections to higher order terms in the expansion (18) are poorly known. A simple way to keep (21) and to include diffraction into the higher terms is to modify (18) as,

$$
\operatorname{Im} \Gamma_{P}(b, s)=\frac{1}{1+D(s)}\left\{1-\exp \left[-(1+D(s)) \operatorname{Im} \gamma_{P}(b, s)\right]\right\}
$$

which is known as quasi-eikonal model [41].

A more consistent way of unitarization suggested in [33 prescribes to use the eikonal expression (18) in terms of the color dipole cross sections and then average it over the transverse separations of all partons. Unfortunately, this procedure is simple only if the dipole cross sections depend quadratically on the separation parameter, which is definitely incorrect for $\sigma_{\bar{q} q}^{N}(R)$ in (四). If it were true one would have $D(s)=1$ which would exceed four times the experimental value. We have tried this unitarization prescription as well and found 
that the results still agree with data pretty well. Nevertheless, we use for further applications Eq. (22) since it explicitly exploits experimental information, correctly reproduces the lowest unitarity correction (21), and is rather accurate within the energy range of interest.

\section{Comparison with forward scattering data}

All the parameters in Eqs. (7), (17) and (22) are known, except the Born cross section $\tilde{\sigma}_{0}$. Although it may be estimated in various models, none of these is sufficiently reliable and we choose to determine it so as to best reproduce the data. As soon as the absolute normalization of the total cross section is fitted at some energy and the parameters $\tilde{\sigma}_{0}$ is fixed, the energy dependence can be predicted. For this comparison we selected the data 42 for $\sigma_{\text {tot }}^{\bar{p} p}$ at $\sqrt{s}=546 \mathrm{GeV}$ as being the most precise. In addition, this energy value is

high enough that we neglect the Reggeon contribution. We calculate $\sigma_{\text {tot }}^{\bar{p} p}$ from

$$
\sigma_{t o t}=2 \int d^{2} b \operatorname{Im} \Gamma(b, s)
$$

using (22) and fix our only unknown parameter at $\tilde{\sigma}_{0}=39.7 \mathrm{mb}$.

Now we are in the position to predict the energy dependence for the total cross sections and compare it with $p p$ and $\bar{p} p$ data. The result is depicted by the dashed curve in Fig. 4 is in a good agreement at high energies, but somewhat off the data at medium high energies. This is not surprising since the (secondary) Reggeon contribution is still missing and this is well known to be important at medium high energies.

In order to improve the description of the data one should, therefore add the contribution of leading Reggeons with intercept $\alpha_{R}(0) \approx 1 / 2$. This should be done directly in the partial elastic amplitude,

$$
\operatorname{Im} \Gamma(s, b)=\operatorname{Im} \Gamma_{P}(s, b)+\Gamma_{R}(s, b)\left[1-\operatorname{Im} \Gamma_{P}(s, b)\right]
$$

The Reggeon term is suppressed by the absorptive corrections which have the same origin as those which slow down the energy dependence of the diffraction cross section mentioned 


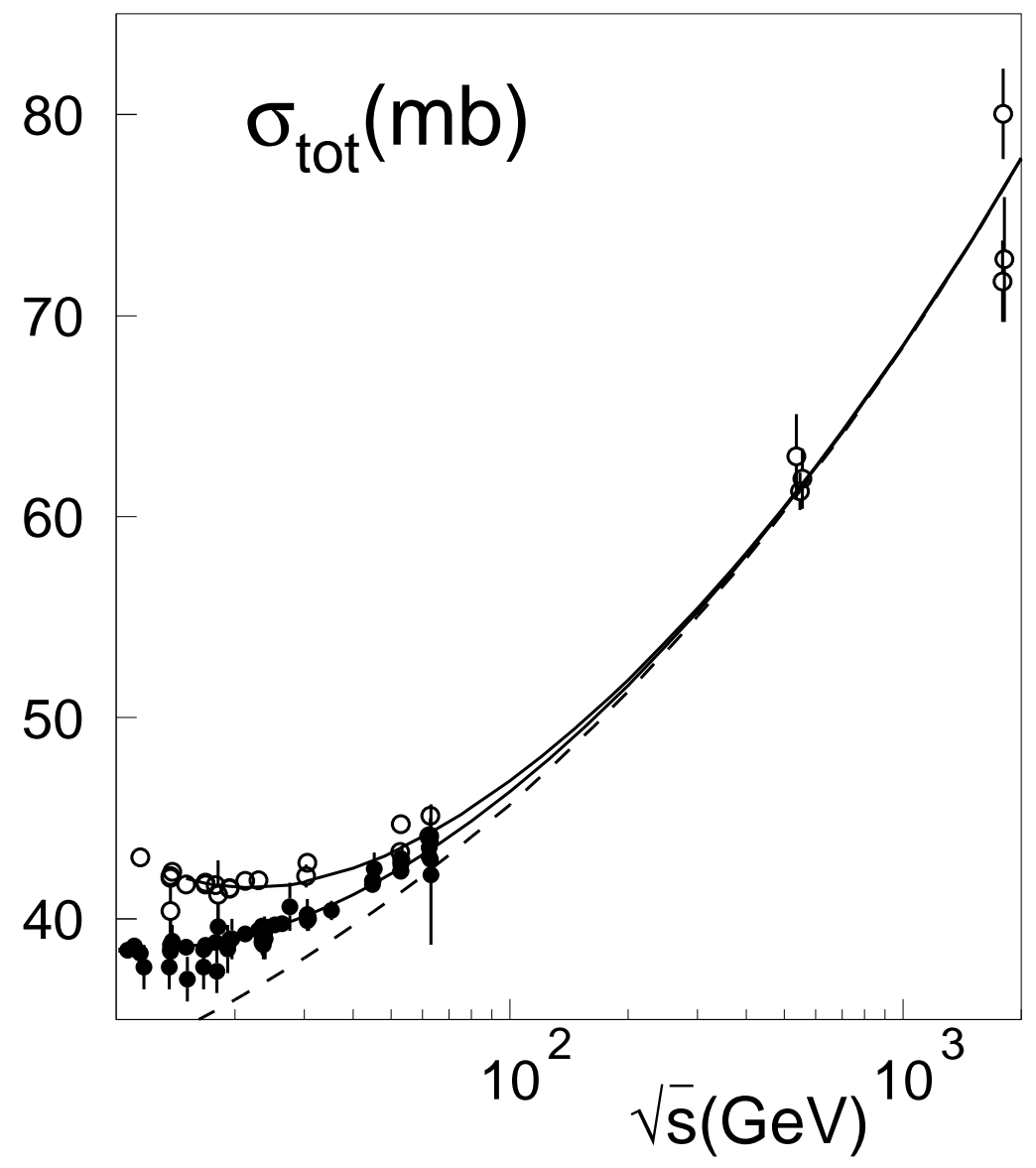

Figure 4: Data for total pp (closed circles) and $\bar{p} p$ ) (open circles) cross sections [43] at $\sqrt{s}>10 \mathrm{GeV}$. The dashed curve shows the predicted energy dependence for the net Pomeron contribution whose normalization is fixed by the $\sqrt{s}=546 \mathrm{GeV}$ data [42]. The solid curves, (bottom for $p p$ and upper for $\bar{p} p$ ), represent the results corrected for Reggeon contribution which is fitted to the data.

above. The Reggeon term is parameterized as,

$$
\operatorname{Im} \Gamma_{R}(s, b)=\frac{\sigma_{R}}{4 \pi B_{R}(s)}\left(\frac{s}{s_{0}}\right)^{\alpha_{R}(0)-1} \exp \left(-\frac{b^{2}}{2 B_{R}(s)}\right),
$$

and $B_{R}=R_{R}^{2}+2 \alpha_{R}^{\prime} \ln s$. We fixed the standard values the parameters $\alpha_{R}(0)=0.5$ and $\alpha_{R}^{\prime}=0.9 \mathrm{GeV}^{-2}$, but fitted $R_{R}^{2}=3 \mathrm{GeV}^{-2}$. We also fitted the normalization factors which are very different for $p p$ and $\bar{p} p$ because of the (approximate) exchange degeneracy. We 
found $\sigma_{R}^{p p}=17.8 \mathrm{mb}$ and $\sigma_{R}^{\bar{p} p}=32.8 \mathrm{mb}$. The result of the fit, shown in Fig. 1 by solid curves demonstrates a good agreement with data. As anticipated, the Reggeon corrections are important only in the ISR energy range and below.

Since $\tilde{\sigma}_{0}$ and all the Reggeon parameters are now fixed, we can predict the slopes of elastic $p p$ and $\bar{p} p$ scattering (both the absolute values and energy dependence). We calculate the slope using the relation,

$$
B_{e l}(s)=\frac{1}{2}\left\langle b^{2}\right\rangle=\frac{1}{\sigma_{t o t}} \int d^{2} b b^{2} \operatorname{Im} \Gamma(b, s) .
$$

Once again, the results shown in Fig. 5 in comparison with data for $p p$ and $\bar{p} p$ scattering demonstrate a good agreement. Although we had some freedom in the choice of the proton formfactor and of the proton charge radius, this affects only the absolute value of the slope. The energy dependence is fully predicted. Since it describes the data well, we correctly predict the effective Pomeron slope $\alpha_{e f f}^{\prime} \approx 0.25 \mathrm{GeV}^{-2}$.

Note that, often, phenomenological fits treat total cross sections and slopes as controlled by different parameters. In these cases, one cannot predict the energy dependent slope even if the total cross section is known.

The radiation of every new gluon leads to an expansion of the gluon cloud by a "step" $\delta\left\langle r^{2}\right\rangle \approx(0.3 \mathrm{fm})^{2}$. Eventually, the initial approximation of a small gluon cloud inside a large hadron will break down. This, however, will happen only at very high energies. The mean number of gluons in a quark $\langle n\rangle=\Delta \ln \left(s / s_{0}\right)$ is quite small, $\langle n\rangle=0.5-0.8$ at ISR, about $\langle n\rangle \approx 1.5$ at $S \bar{p} p S$ and reaches $\langle n\rangle \approx 2$ at the Tevatron. Therefore the mean radius of a constituent quark is still rather small and our approximation remains quite valid and we should expect it to break down only at very high energies which are well beyond the range of present accelerators. Of course the radius of an constituent quark, i.e. the radius of the gluon cloud depends also on a reference frame, e.g. in the c.m. it is twice as small as in the rest frame of the target. 


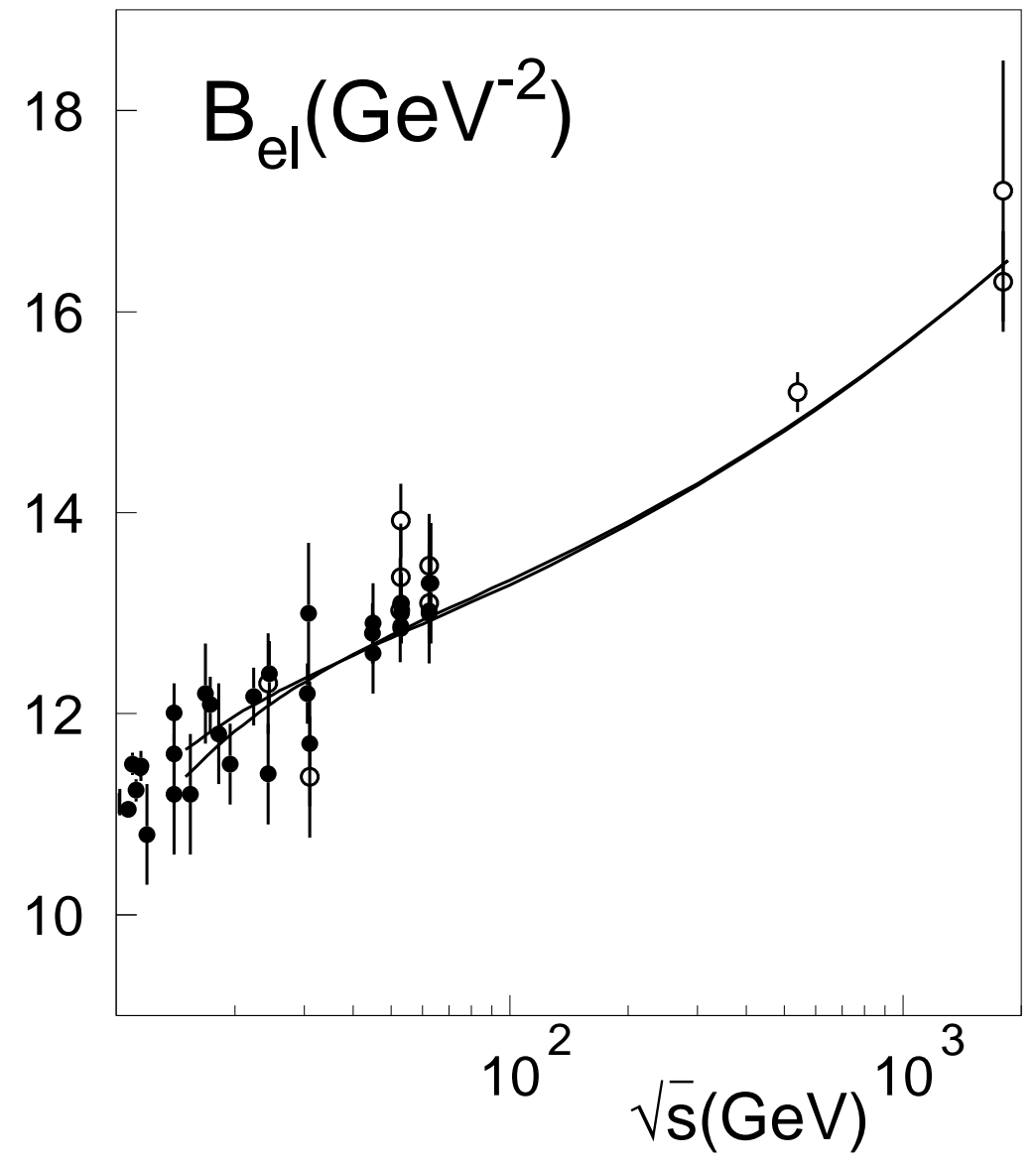

Figure 5: Data for the elastic slope [44] and our predictions. The upper and bottom curves and, correspondingly, the open and full circles belong to $\bar{p} p$ and $p p$, respectively.

\section{Elastic scattering data analyzed in the impact pa- rameter}

The partial amplitude (22) has nontrivial $s$ - and $b$-dependences. It is nearly energy independent for central collisions, but steeply grows with energy on the periphery as it was first found in the analysis of the data by Amaldi and Schubert [29]. These properties are averaged out and hidden in the total or differential elastic cross sections. To extract information about the shape of the partial elastic amplitude in the impact parameter representation from the 
data on elastic $p p$ and $\bar{p} p$ scattering, we follow the procedure used in [29]. However, to make the analysis less model dependent we fit differential elastic cross section data independently at each energy, thus, no model for energy dependence is involved. The geometrical scaling model used in [29] assumes that the total cross section is proportional to the slope of the elastic differential cross section, $\sigma_{\text {tot }}^{p p}(s) \propto B^{p p}(s)$. This relation is, however, a result due to the accidental closeness of the Regge model parameters characterizing the energy dependence of the cross section, $\sigma_{\text {tot }}^{p p}(s) \propto s^{\Delta} \approx 1+\Delta \ln s$ and of the slope, $B^{p p}(s)=B_{0}^{p p}+2 \alpha_{P}^{\prime} \ln s$, where $\alpha_{P}^{\prime} \approx 0.25 \mathrm{GeV}^{-2}$ and $B_{0}^{p p} \approx 7.5 \mathrm{GeV}^{-2}$. Indeed, the effective Pomeron intercept $\Delta=\alpha_{P}(0)-1 \approx 0.08$ is close to the ratio $2 \alpha_{P}^{\prime} / B_{0}^{p p} \approx 0.067$. Obviously, geometrical scaling may occur only in a restricted energy range (namely, in the ISR energy range used in [29]) and it had been predicted [2] to break down at higher energies. This was confirmed later by the $S p \bar{p} S$ and Tevatron data.

Since we are interested in the Pomeron part of the elastic amplitude, the data for differential cross section of elastic $p p$ and $\bar{p} p$ scattering selected for the analysis cover the wide range of high energies including $I S R$ ], and $S p \bar{p} S$ [45]. We do not include the data from the Tevatron since they are available only in too narrow a range of $t$ which is not sufficient for Fourier transformation. We parameterize the imaginary and real parts of the elastic scattering amplitude in a model independent way as,

$$
\begin{aligned}
& \operatorname{Im} f(t)=\sum_{i=1}^{3} a_{i} e^{b_{i} t} \\
& \operatorname{Re} f(t)=\sum_{i=1}^{2} c_{i} e^{d_{i} t},
\end{aligned}
$$

where $a_{i}, b_{i}, c_{i}, d_{i}$ are parameters to be fitted. The amplitudes are related to the cross sections as,

$$
\begin{gathered}
\frac{d \sigma}{d t}=[\operatorname{Re} f(t)]^{2}+[\operatorname{Im} f(t)]^{2} ; \\
\sigma_{t o t}=4 \sqrt{\pi} \operatorname{Im} f(0) .
\end{gathered}
$$

\footnotetext{
${ }^{2}$ We rely upon the relative normalizations of differential cross sections measured in different $t$-intervals at the same energy given in 29]
} 
To make the normalization of the differential cross section data more reliable, first we perform a common fit of the $p p$ and $\bar{p} p$ total cross sections with the same Pomeron part as function of energy. Then we adjust the normalizations of the differential elastic cross section data to the optical points, i.e. demand that $4 \sqrt{\pi} \sum a_{i}=\sigma_{\text {tot }}$ at each energy. The data [46] for $\rho(s)$ ratio of the real to the imaginary parts of the amplitudes at $t=0$ were also involved in the analysis. We fit these data by a smooth energy dependence and demand then $\sum c_{i}=\rho \sum a_{i}$ for each energy included in the analyses of differential cross sections. We performed two variants of fit,

- variant $I$ : both $c_{1}$ and $c_{2}$ are used as free parameters in the fit;

- variant II: $c_{2}=0$ in (28).

The data in the fit and the results in variant $I$ are depicted in Fig. 6 .

As soon as the parameters in (27) and (28) are found we can calculate the partial amplitude in the impact parameter representation at each energy ,

$$
\Gamma(b)=\frac{1}{2 \pi^{3 / 2}} \int d^{2} q e^{i \vec{q} \cdot \vec{b}} f\left(-q^{2}\right)
$$

where $\vec{q}$ is the transverse component of the momentum transfer, $t \approx-q^{2}$. It is normalized according to (23).

A few examples of our results for $\operatorname{Im} \Gamma(b)$ corresponding to variant $I$ (full points) and variant II (open points) are shown in Fig. 7 with spacing $0.2 \mathrm{fm}$ in the impact parameter and for a few energies. The errors are calculated using the error matrix resulting from the fit.

One can see that at $b=0$ the amplitude nearly saturates the unitarity limit and hardly changes with energy, while at larger impact parameters the amplitude grows quite substantially.

Our predictions including the Pomeron contribution and Reggeon part are compared with the data in Fig. 17. The Reggeons shown by dashed curves are calculated for $p p$ and $\bar{p} p$ interactions for ISR and $\mathrm{S} p \bar{p} \mathrm{~S}$ data respectively. Their contribution is quite a small fraction 


\section{$\mathrm{d} \sigma / \mathrm{dt}(\mathrm{mb}) /(\mathrm{GeV})^{2}$}
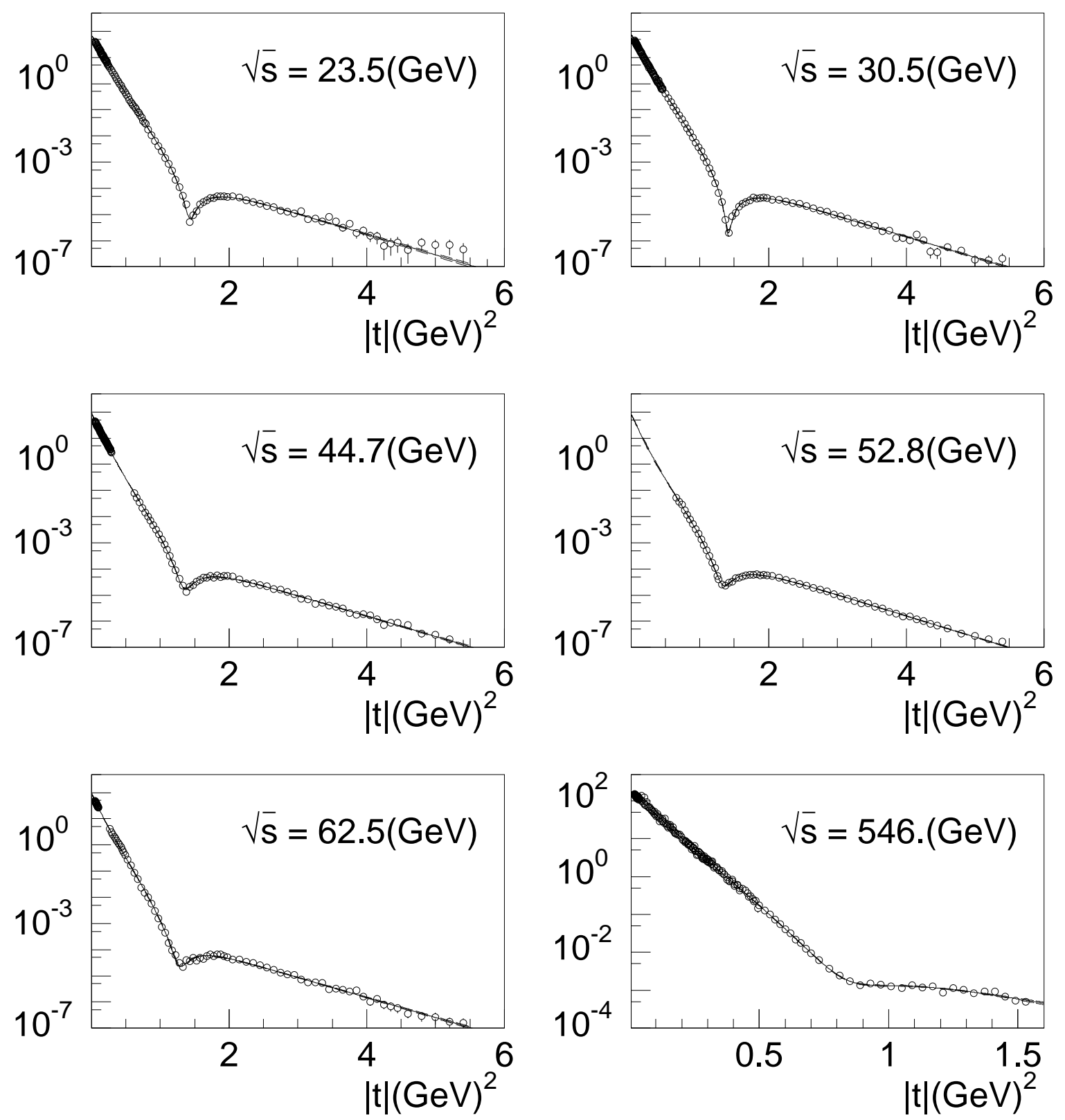

Figure 6: The differential cross sections of elastic $p p$ and $\bar{p} p$ scattering at different energies. The first five panels show the $p p$ data from ISR 29], the last one the $\bar{p} p$ data [45] from $S p \bar{p} S$. The curves show our fit Eqs. (27)-(29) in variant $I$. 
of the full partial amplitude represented by the solid curves. The agreement between the data and our predictions is remarkably good, especially if we recall that the Pomeron part

\section{$\operatorname{lm} \Gamma(\mathrm{b})$}

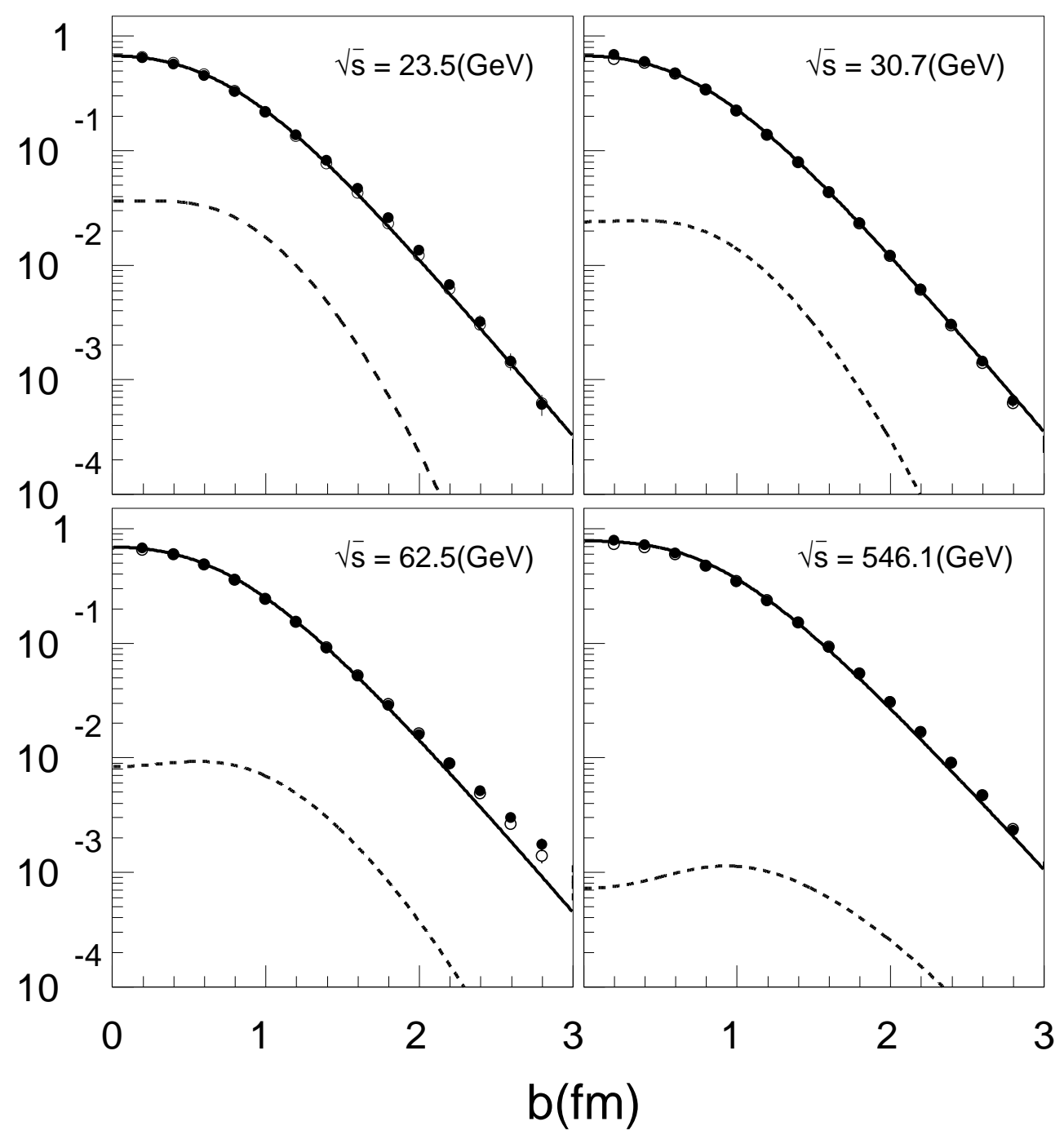

Figure 7: The imaginary part of the partial amplitude $\operatorname{Im} \Gamma(b)$ as function of the impact parameter at different energies. The first three panels correspond to the ISR, the last one to the $S p \bar{p} S$ data. The curves show our theoretical prediction with Eq. (22) using the parameters given in Table 1 and obtained fitting the $t=0$ data (total cross sections and slopes). 
has no free parameters, except one, $\tilde{\sigma}_{0}$, adjusted to the total cross section measured at one energy $\sqrt{s}=546 \mathrm{GeV}[42]$. Both the predicted shape of the partial amplitude and its energy development are confirmed by the data.

\section{Pomeron trajectory in the impact parameter space}

The partial elastic amplitude rises with energy faster for peripheral than for central collisions. The energy dependence of $\operatorname{Im} \Gamma(b, s)$ at different values of the impact parameter is shown in Fig. 8 for variant I. One can see by the eye that the upper curves corresponding to central

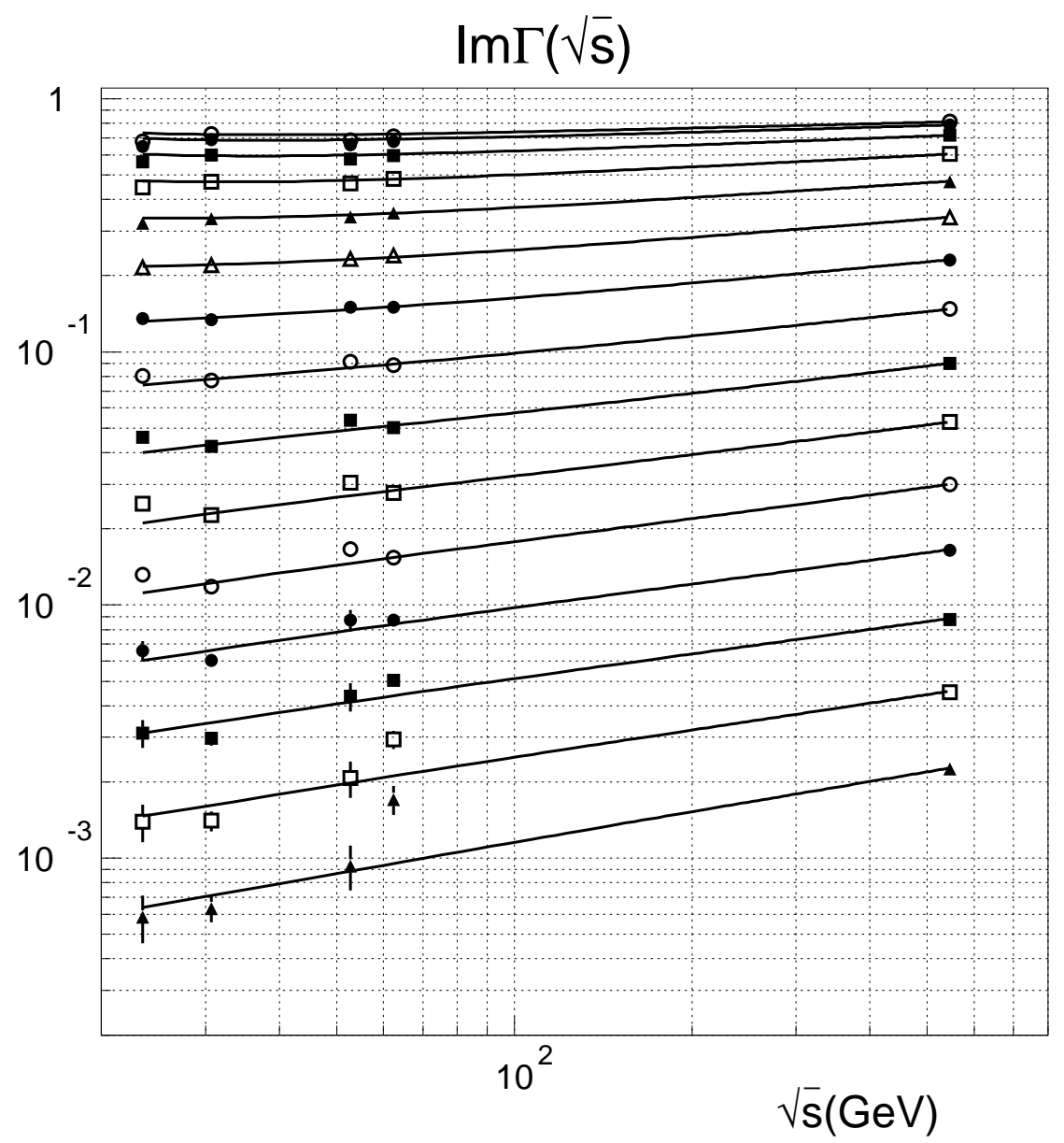

Figure 8: $\operatorname{Im} \Gamma(b)$ plotted at various values of $b=0.0,0.2,0.4, \ldots 2.8 \mathrm{fm}$ as function of energy. The values and error bars correspond to Fig. 7 . The lines correspond to the fit with power dependence on energy. 
collisions are nearly horizontal, while the bottom ones representing peripheral collisions rise steeply with energy. The curves show the results of the fit to the data for energy dependence of the partial amplitude at different values of the impact parameter by the expression

$$
\operatorname{Im} \Gamma(b, s)=\Gamma_{0} s^{\Delta(b)}+\operatorname{Im} \Gamma_{R}(b, s),
$$

in which the Reggeon term (25) is calculated with the parameters fixed by the fit to total cross section data. The exponent $\Delta(b)$ varies with the impact parameter and is fitted to the data for what concerns its energy dependence in each bin of $b$ as it is shown in Fig. 8. We ignored the data at $\sqrt{s}=62 \mathrm{GeV}$ since they are too much off the smooth interpolation of the data at lower and higher energies. The results of the fit for $\Delta(b)$ are plotted in Fig. 9 as a series of black points corresponding to each of the lines in Fig. 8. Open points show the results of the fit corresponding to variant II. The error bars are determined by the error matrix of the fit. Note that these values of $\Delta(b)$ correspond to the Pomeron contribution since the Reggeon part is sorted out.

Although $\Delta(b)$ is very small $\sim 0.03$ for central collisions, $(i . e$ near $b=0$ ), it increases dramatically (nearly by one order of magnitude) at large $b$. Thus, the data show that the energy dependence of the total cross section originates mainly from peripheral interactions. This confirms the observation of [29].

The systematic uncertainty of our analysis is related mainly to the choice of parameterization for the elastic amplitude. The difference between variants I and II can be treated as a characteristic estimate of this uncertainty. There is no significant difference between the two solutions.

Our predictions plotted as solid curves in Fig. (7) can also be translated into values for the effective exponent $\Delta(b)$,

$$
\Delta_{e f f}(b)=\frac{d \ln \left[\Gamma_{P}(s, b)\right]}{d \ln s},
$$

using the theoretical amplitude (22) with the same parameters already determined. The results are shown as a solid curve in Fig. 9. It agrees well with the data. 


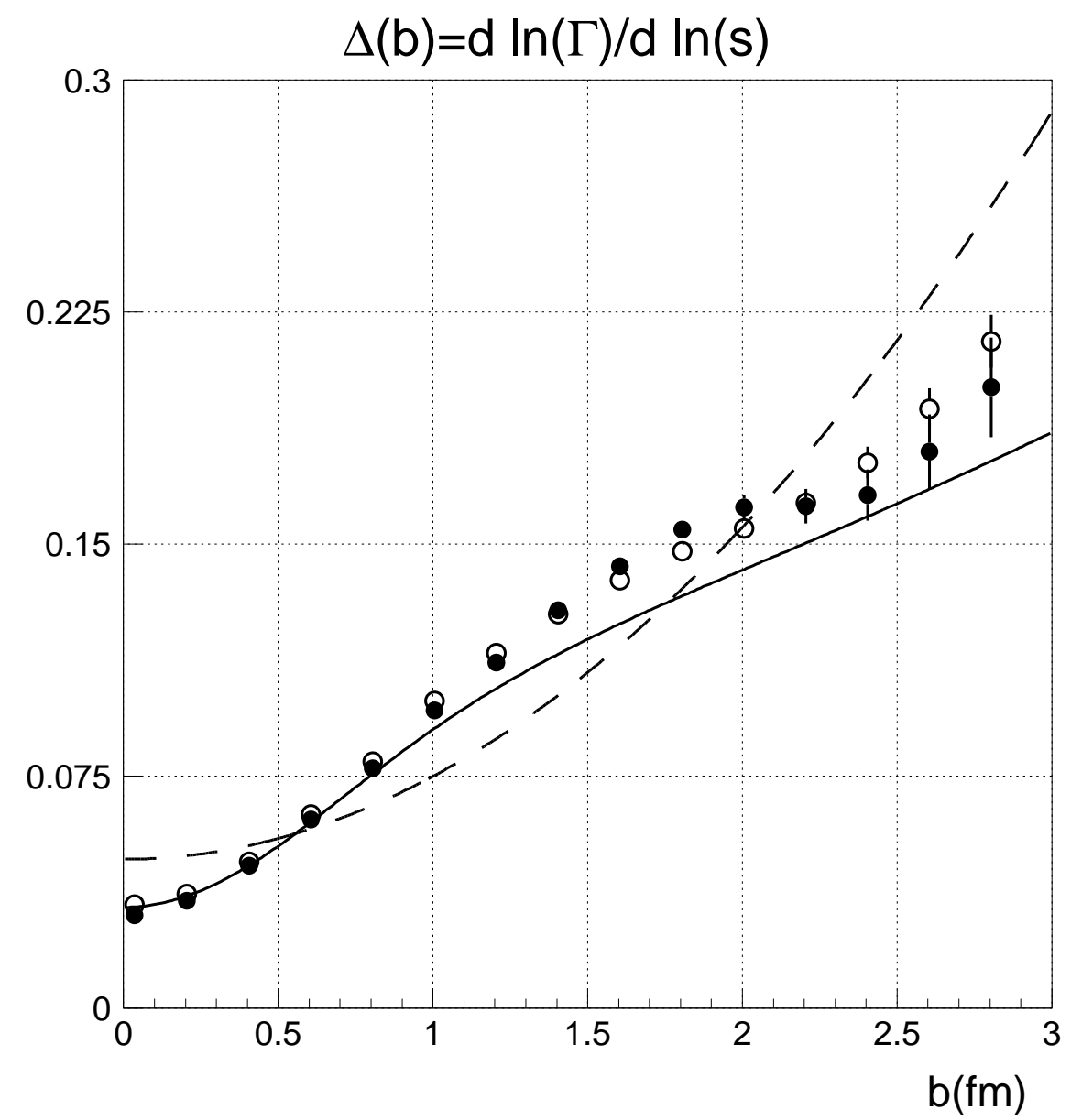

Figure 9: The exponent $\Delta(b)$ found by the fit to each point of Fig. 8 with power dependence on energy at each value of $b$. The black and open points correspond to the fits with parameterizations I and II respectively. Our predictions with Eq. (33) are shown by solid curve. The dashed curve demonstrates prediction of a single Regge pole model without any unitarity corrections.

The dashed curve shows the prediction of the simplest parameterization [7, 8] for the elastic amplitude with power $s$ - and exponential $t$ - dependences for both the Pomeron and Reggeon terms. Although the unitarity corrections are neglected, this parameterization is indeed quite successful in describing total cross sections and elastic slopes $[8]$. One can see, however, that its agreement with the data in Fig. 9 is quite poor. It overshoots the 
data for central (lack of unitarization) and peripheral collisions and has quite a different $b$-dependent shape. Nevertheless, one should not interpret the smallness of $\Delta$ at $b=0$ as a manifestation of saturation of unitarity.

\section{Summary, discussion and outlook}

We present the first successful quantitative dynamical description of small angle elastic scattering of light hadrons. The key points of our approach are,

- The data for diffractive gluon radiation (large mass diffraction) demand a small transverse separation $r_{0} \approx 0.3 \mathrm{fm}$ between the radiated gluon and the valence quark [12, 13].

- A new regime is found which allows explicit calculations: the gluon clouds of valence quarks are much smaller than the hadronic size, $r_{0}^{2} / R_{h}^{2} \ll 1$. This is a different limiting case compared to DIS where the gluon cloud is much larger than the $\bar{q} q$ separation.

- The interference between amplitudes of gluon radiation by different quarks is suppressed as $\exp \left(-R_{h}^{2} / r_{0}^{2}\right)$ leading to an additivity of the valence quarks in the part of the total cross section related to gluon radiation.

- Since gluon radiation is controlled by the semi-hard scale $Q \sim 2 / r_{0}$ it can be evaluated perturbatively. The radiation cross section is suppressed by the small factor $r_{0}^{2} \approx 1 \mathrm{mb}$, but steeply rises with energy $\propto s^{\Delta}$, where $\Delta$ is given by Eq. (14).

- The part of the total cross section $\tilde{\sigma}_{0}$ related to soft collisions without excitation of the valence quarks is large since it is controlled by the large hadronic radius. It is independent of energy and may cause deviation from quark additivity.

- The two-scale structure $\left(r_{0}\right.$ versus $\left.R_{h}\right)$ of light hadrons unavoidably leads to the specific form (11) of energy dependence for the total cross section. The terms with and without gluon radiations are governed by different scales and cannot match in order to exponentiate into a common factor $s^{\Delta}$ for the whole cross section. 
- While the second, energy dependent term in Eq. (1) can be evaluated perturbatively, none of current models can estimate reliably the first constant term $\tilde{\sigma}_{0}$. We treat it as a free parameter and fix it by the normalization of total cross sections. Then we predict the energy dependence of the total cross section and the forward elastic slope in good accord with data.

- As a further rigorous test of the model, we perform a model-independent analysis of available high-energy data for the elastic differential cross sections and extract the partial amplitudes in impact parameter representation. Our model describes well the observed $b$ - and $s$ - dependences of the partial amplitude.

- We also extract the Pomeron trajectory $\alpha_{P}(b)=1+\Delta(b)$ in the impact parameter representation. $\Delta(b)$ is very small at $b=0$ as a result of unitarity saturation, but rises by an order of magnitude for peripheral collisions, in good accord with our predictions.

Concluding, the strong interaction of radiated gluons is vital for present approach. It squeezes the gluon clouds of valence quarks and allows to apply perturbative QCD to calculation of the radiation cross section. It is worth emphasizing that it is not legitimate to mimic these nonperturbative effects introducing an effective gluon mass $m_{G} \sim 0.7 \mathrm{GeV}$ as is frequently done in the literature. Indeed, only the light-cone gluons interact nonperturbatively during their long lifetime. However, the t-channel Coulomb gluons cannot be treated on the same footing as the light-cone ones, their lifetime is always short. These gluons are massless and can propagate far away. To incorporate the confinement one should assign only a small effective mass $m_{G} \sim \Lambda_{Q C D}$ to the t-channel gluons. Making these t-channel gluons as heavy as those on the light-cone would suppress the factor $C$ in (9) and the term $\sigma_{1}$ in (11) by nearly order of magnitude in contradiction with data.

Note that our results explain the surprisingly high effective Pomeron intercept $\Delta \approx 0.2$ observed in diffractive DIS. It is known that diffraction is dominated by soft interactions even at high $Q^{2}$ and one could expect about twice smaller value. However, large mass 
diffraction related to diffractive gluon radiation corresponds to the second term in Eq. (9), i.e. value of $\Delta$ given by (14).

Although data for diffractive dissociation in soft hadronic collisions were used to fix the strength of the nonperturbative gluon interaction, one can further test the model performing a similar analysis of data for the differential cross section of single diffraction in the impact parameter representation. Such an analysis is in progress and will be published elsewhere.

In this paper we concentrate on calculation of the elastic hadronic amplitude related via unitarity to inelastic processes like gluon radiation. It is natural to extend the test of the model comparing directly to data for multiparticle production. In particular, the AGK cancelation [3] of unitarity corrections leads to the inclusive cross section in central region of rapidities rising with energy as $s^{\Delta}$ where $\Delta$ has its genuine value not disturbed by unitarity corrections. Such an analysis of data performed in [47] has led to a surprisingly similar conclusions as ours. Namely, (i) the data cannot be described by the energy dependence $\propto s^{\Delta}$ in the whole energy range, but demand an additional constant term; (ii) the fit to data resulted in $\Delta=0.17$, exactly what is predicted by our calculations.

Note that the model suggested in [48] which describes multiparticle production in terms of energy independent string fragmentation and rising with energy mini-jet contribution also goes along with the basics of our model.

Acknowledgments: We are thankful to Jörg Hüfner, Hans-Jürgen Pirner, Andreas Schäfer and Sasha Tarasov for inspiring and helpful discussions. We are grateful to Anatoli Likhoded who informed us about the analysis of data for inclusive cross sections performed in Ref. 477 which perfectly agree with our results. This work was partially supported by the grant INTAS-97-OPEN-31696, by the European Network: Hadronic Physics with Electromagnetic Probes, Contract No. FMRX-CT96-0008 and by the INFN and MURST of Italy.

\section{References}


[1] B.Z. Kopeliovich, I.K. Potashnikova, B. Povh and E. Predazzi, Phys. Rev. Lett. 85 (2000) 507.

[2] M.I. Dubovikov, B.Z. Kopeliovich, L.I. Lapidus and K.A. Ter-Martirosyan, Nucl. Phys. B123 (1977) 147.

[3] A.V. Abramovsky, V.N. Gribov and O.V. Kancheli, Yad. Fiz. 18 (1973) 595.

[4] A. Capella, A. Kaidalov, C. Merino and J. Tran Thanh Van, Phys. Lett. B337 (1994) 358.

[5] M. Bertini, M. Giffon and E. Predazzi, Phys. Letters B349 (1995) 561.

[6] P. Desgrolard, M. Giffon, E. Martynov and E. Predazzi, DFTT-66-99 (Dec. 1999), hep-ph 0001149 (to appear on Eur. Phys. J.).

[7] A. Donnachie and P. Landshoff, Nucl. Phys. B303 (1988) 634.

[8] Review of Particle Properties, Phys. Rev. D54 (1996) 1.

[9] E.A. Kuraev, L.N. Lipatov and V.S. Fadin, Sov. Phys. JETP 44 (1976) 443 ; 45 (1977) 199; Ya.Ya. Balitskii and L.I. Lipatov, Sov. J. Nucl. Phys. 28 (1978) 822; L.N. Lipatov, Sov. Phys. JETP 63 (1986) 904.

[10] Yu.L. Dokshitzer, V.A. Khoze, A.H. Mueller and S.I. Troyan, Basics of Perturbative QCD, Editions Frontieres, ADAGP, Paris 1991.

[11] B. Badelek, K. Charchula, M. Kravczyk and J. Kwiecinski, Rev. Mod. Phys. 64 (1992) 927.

[12] B.Z. Kopeliovich, A. Schäfer and A.V. Tarasov, Phys. Rev. D62 (2000) 054022 (hep-ph/9908245).

[13] F. Hautmann and D.E. Soper, Color transparency in deeply inelastic diffraction, hep-ph/0008224 
[14] V.M. Braun, P. Górnicki, l. Mankiewicz and A. Schäfer, Phys. Lett. B302 (1993) 291.

[15] M. D'Elia, A. Di Giacomo and E. Meggiolaro, Phys. Lett. B408, 315 (1997).

[16] T. Schäfer, E.V. Shuryak, Rev. Mod. Phys. 70, 323 (1998).

[17] E.V. Shuryak, Toward the non-perturbative description of high energy processes, hep-ph/0001189.

[18] F.E. Low, Phys. Rev. D12 (1975) 163.

[19] S. Nussinov, Phys. Rev. Lett. 34 (1975) 1986.

[20] J.F. Gunion and D.E. Soper, Phys. Rev. D15 (1977) 2617.

[21] D. Kharzeev and E.M. Levin, Nucl.Phys. B578 (2000) 351.

[22] D. Kharzeev, Yu. Kovchegov and E.M. Levin, QCD Instantons and the Soft Pomeron, hep-ph/0007182.

[23] E. Gotsman and S. Nussinov, Phys. Rev. D 22 (1980) 624.

[24] H.G. Dosch, Phys. Lett. B190, 177 (1987); H.G. Dosch and Yu.A. Simonov, Phys. Lett. B205, 339 (1988).

[25] H.G. Dosch, T. Gousset, G. Kulzinger and H.J. Pirner, Phys. Rev. D55, 2602 (1997)

[26] J. Hüfner and B. Povh, Phys. Rev. D46 (1992) 990.

[27] B. Povh, Hadron Interactions - Hadron Sizes, Advances in Nuclear Dynamics 4, ed. W. Bauer and H.-G.Ritter Plenum Press.N.Y. page 267 (hep-ph/9806379).

[28] B. Z. Kopeliovich, B. Povh and E. Predazzi, Phys. Lett. B405 (1997) 361.

[29] U. Amaldi and K.R. Schubert, Nucl. Phys. B166 (1980) 301. 
[30] Al.B. Zamolodchikov, B.Z. Kopeliovich and L.I. Lapidus, Sov. Phys. JETP Lett. 33 (1981) 612.

[31] C. Bourrely et al., Phys. Rev. D26 (1982) 1781.

[32] B.Z. Kopeliovich, Soft Component of Hard Reactions and Nuclear Shadowing (DIS, Drell-Yan reaction, heavy quark production), in proc. of the Workshop Hirschegg'95: Dynamical Properties of Hadrons in Nuclear Matter, Hirschegg, January 16-21,1995, ed. by H. Feldmeier and W. Nörenberg, Darmstadt, 1995, p. 102 (hep-ph/9609385).

[33] B.Z. Kopeliovich, N.N. Nikolaev and I.K. Potashnikova, Phys. Lett. 209B (1988) 335; Phys. Rev. D39 (1989) 769.

[34] V.N. Gribov, Eur. Phys. J. C10 (1999) 71; ibid C10, 91 (1999).

[35] C. Ewerz, Eur. Phys. J. C13 (2000) 503.

[36] V.A. Khoze, A.D. Martin and M.G. Ryskin, hep-ph/0007359

[37] Yu. Dokshitzer, G. Marchesini, B. Webber, Nucl. Phys. B469, 93 (1996).

[38] V.N. Gribov, Sov. Phys. JETP 57 (1969) 1306.

[39] K. Goulianos, J. Montanha, Phys. Rev. D59 (1999) 114017.

[40] S. Erhan and P.E. Schlein, Phys. Lett. B427 (1998) 389.

[41] A.B. Kaidalov, Phys. Rept. 50, 157 (1979).

[42] F. Abe et al., Phys. Rev. D50, 550 (1993).

[43] C. Caso et al, The Eur. Phys. J. C3, 1 (1998).

[44] U. Amaldi et al., Phys. Lett. 36B (1971) 504; 66B (1977) 390; M. Ambrosio et al., Phys. Lett. 115B (1982) 495; N. Amos et al., Phys. Lett. 128B (1983) 343; 
Nucl. Phys. B262 (1985) 689; Phys. Rev. Lett. 61 (1988) 525; Phys. Rev. Lett. 63 (1989) 2784; V. Apokin et al., Sov. J. Nucl. Phys. 25 (1977) 51; V. Bartenev et al., Phys. Rev. Lett. 29 (1972) 1755; G. Beznogikh et al., Nucl. Phys. B54 (1973) 78; M. Bozzo et al., Phys. Lett. 147B (1984) 385; A .Breakstone et al., Nucl. Phys. B248 (1984) 253; R.E. Breedon et al., Phys. Lett. 216B (1989) 459; C. Bromberg et al., Phys. Rev. D15 (1977) 64; J.P. Burq et al., Phys. Lett. 109B (1982) 124; R.L. Cool et al., Phys. Rev. D24 (1981) 2821; D. Favart et al., Phys. Rev. Lett. 47 (1981) 1191.

[45] R. Battiston et al., Phys. Lett. 127B (1983) 472; M. Bozzo et al., Phys. Lett. 147B (1984) 385; 155B (1985) 197; D. Bernard et al., Phys. Lett. 198B (1987) 583; G. Arnison et al., 128B (1983) 336.

[46] U. Amaldi et al., Nucl. Phys. 166B (1980) 301; C. Augier et al., Phys. Lett. 316B (1993) 448; N. Amos et al., Phys. Rev. Lett. 68 (1992) 2433.

[47] A.K. Likhoded and O.P. Yushchenko, Int. J. Mod. Phys. A6 (1991)913; A.K. Likhoded, V.A. Uvarov, P.V. Chliapnikov, Phys. Lett. B215 (1988) 417

[48] X.-N. Wang, Phys. Rept. 280 (1997) 287 